\title{
Effective mixing and aeration in a bioreactor with Taylor vortex flow
}

\author{
Hiroshi YOKOYAMA*, Yuu HIROSE** and Akiyoshi IIDA* \\ *Department of Mechanical Engineering, Toyohashi University of Technology \\ 1-1 Hibarigaoka, Tempaku, Aichi 441-8580, Japan \\ E-mail: h-yokoyama@me.tut.ac.jp \\ **Department of Environmental and Life Sciences, Toyohashi University of Technology \\ 1-1 Hibarigaoka, Tempaku, Aichi 441-8580, Japan
}

Received 5 July 2016

\begin{abstract}
In order to clarify the effects of the behaviors of the bubbles of aeration on the productivity of biomass, experiments including cultivation and computation were performed utilizing a bioreactor with a Taylor vortex flow in the annular gap of concentric cylinders. The shape of the inner cylinder and the position of the aeration were changed to vary the floating speed of the bubbles. The flow velocity was measured by Particle Image Velocimetry and the time taken for the floating of the bubbles from the lower wall to the upper surface of the suspension was also measured along with prediction by Large Eddy Simulations. The cultivation of cyanobacteria was also performed. Attaching spiral fins to the inner cylinder induces downward velocity in the region close to the outer cylinder, where a large-scale vortex replaces the original three vortical structures generated in the case of a simple circular inner cylinder. As a result, the time taken for the floating of the bubbles becomes longer when the aeration was performed at a position close to the outer cylinder. When cultivation was performed in these conditions, the cyanobacteria were found to have developed up to a high content and that their growth rate was also high. The present results indicate that the behaviors of the bubbles of aeration are related to the productivity of the biomass.
\end{abstract}

Key words : Bioreactor, Taylor vortex flow, Cultivation, Algae, Particle image velocimetry, Bubbles, Large eddy simulation

\section{Introduction}

Recently, algae have attracted an increasing attention as a source for sustainable food, feed, and biomass (biofuels), but cost of cultivation is a problem for practical production of these algae-based materials. To reduce the cost, the algae themselves need to be cultivated efficiently. As reviewed by many authors (Eriksen, 2008, Jassen et al., 2003, Grobbelaar, 2000, and Pulz, 2001), different culturing techniques for photoautotrophic growth have been investigated. To increase the productivity of the algal biomass, we need greater knowledge about the growth characteristics of algae and to optimize cultivation parameters such as mixing and aeration. It was found that high gas flow velocities entering the photobioreactor can cause high shear rates and cell death (Barbosa et al., 2003).

A bioreactor consisting of inner rotating and outer fixed cylinders, as shown in Fig. 1, was used for the cultivation of algae by some authors (Miller et al., 1964, Kliphuis et al, 2010, and Kawai, 2010), where the algal cell suspensions were placed in the annular space between the two cylinders. With comparison to bioreactors with impellers, the generation of local high shear stress can be expected to be prevented.

The fluid-dynamical research of the flow between these two cylinders has been investigated by various authors (Benjamin, 1978, 1979, 1981, Coles, 1965, and Gorman and Swinney, 1982) since Taylor (Taylor, 1923), and is referred to as Taylor vortex flows, where multiple vortical structures are generated. The experiments by Nakamura $e t$ al. (Nakamura et al., 1988) showed that an odd number of vortices basically occur if the upper boundary of the fluid is a free surface while an even number of vortices occur in the case of a fixed wall. Recently, direct numerical simulations have been performed for the turbulent Taylor vortex flow, and it has been clarified that coherent fine vortical structures are included in large-scale vortices (Bilson and Bremhost, 2007, Dong, 2007, Fukushima, 2010). 
The experiments by Miller et al. (Miller et al., 1964) regarding a bioreactor with the Taylor vortex flow indicated that the rate of photosynthesis increases with an increasing rotor speed. Also, Kliphuis et al. (Kliphuis et al., 2010) investigated the effects of the density of algae in the Taylor vortex flows on productivity. Also, it has been confirmed by Kawai (Kawai, 2010) that cell death is negligible in the range below the Reynold number based on the gap, $d$, between cylinders of $R e=30000$, where the flow field was also measured by an ultrasonic Doppler velocity profiler. However, to the knowledge of the authors, the effects of behaviors of the bubbles of aeration on the growth of the algae have not yet been sufficiently clarified.

In this paper, the effects of adding fins on the inner cylinder on the flow and bubbles in the above-mentioned bioreactor with the Taylor vortex flow are mainly investigated, and the position of the inlet for the aeration is also changed, where the floating speed of the bubbles is varied. Moreover, the effects of those fins and the way of aeration on the growth of the cyanobacteria are investigated, and the relationship between the behaviors of bubbles and the growth of the algae is discussed.
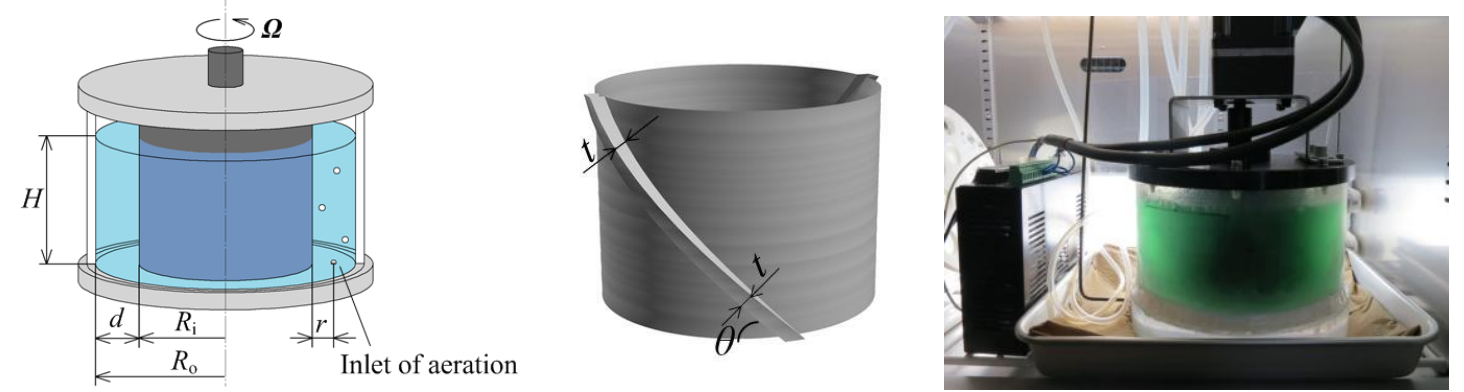

Fig. 1 Bioreactor with Taylor vortex flow in concentric cylinders utilized in the present experiments. The left figure shows illustrations of the bioreactor. The middle figure shows inner cylinder with two spiral fins of the present bioreactor. The right figure shows a picture taken during the cultivation of cyanobacteria (synechocystis sp PCC 6803).
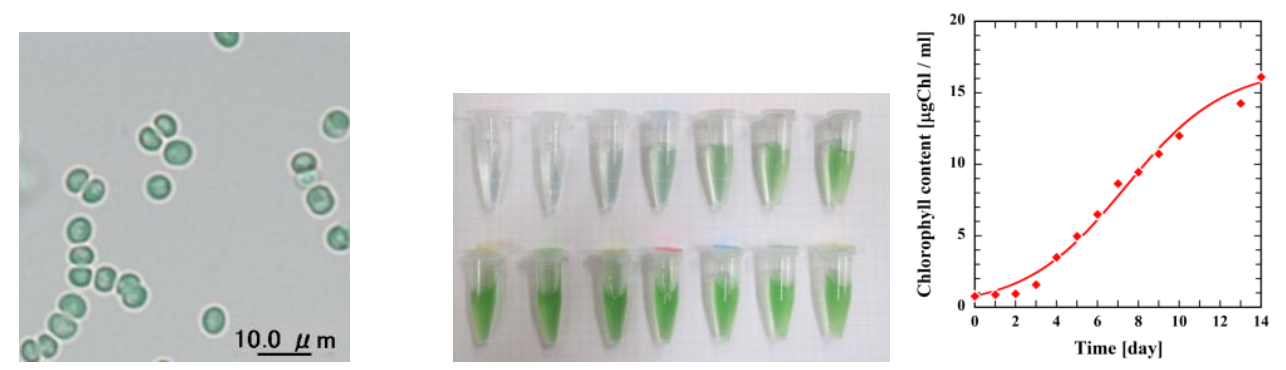

Fig. 2 Cyanobacteria (Synechocystis sp. PCC 6803). The left figure shows the cells of the cyanobacteria. The middle figure shows the concentration variation of the cyanobacteria during the cultivation (no rotation), where sampling was performed every 24 hours. The right figure shows the variation of chlorophyll contents during a cultivation.

Table 1 Parameters of the present bioreactor with Taylor vortex flow.

\begin{tabular}{c|c|c|c|c}
\hline \hline$R_{\mathrm{o}}[\mathrm{mm}]$ & $R_{\mathrm{i}}[\mathrm{mm}]$ & $d[\mathrm{~mm}]$ & $\Gamma$ & $R e$ \\
\hline 75 & 50 & 25 & 3.0 & 0 (no rotation), 4000
\end{tabular}

\section{Methodologies}

\subsection{Bioreactor}

The bioreactor shown in Fig. 1 was used, where the outer cylinder is made of transparent acrylic in order to measure the flow field and behavior of the bubbles by a high-speed camera. Also, the outer cylinder, which was made of thermally resistant polycarbonate, was used for the cultivation because sterilization at a high temperature is necessary before main cultivation.

As shown in Table 1, the radius of the inner cylinder is $R_{\mathrm{i}}=50 \mathrm{~mm}$ and that of the outer cylinder is $R_{\mathrm{o}}=75 \mathrm{~mm}$. The aspect ratio of the height of the suspensions, $H$, to the gap between cylinders, $d$, is $\Gamma=3.0$. The condition of the upper boundary of suspension is a free surface. The rotation speed was adjusted to set the Reynolds number based on the rotation speed of the inner cylinder, $U_{0}$, and the gap between the cylinders to be $R e \equiv U_{0} d / v=4000$ and Taylor 
number $T a \equiv\left(d / R_{\mathrm{i}}\right)^{0.5} U_{0} d / v=2830$, where $v \equiv \mu / \rho$ is a dynamic viscosity of $0.893 \times 10^{-6} \mathrm{~m}^{2} / \mathrm{s}$ (viscosity: $\mu=0.890 \times 10^{-3}$ Pa.s, density: $\rho=0.997 \times 10^{3} \mathrm{~kg} / \mathrm{m}^{3}$ ) based on a water at $25^{\circ} \mathrm{C}$. According to the direct numerical simulation (He et al., 2007), the fine coherent turbulent vortices occur in the Taylor vortex flow at $R e>3000$. The present flow is estimated to be a weakly turbulent flow. To clarify the effects of the rotation, experiments without rotation $(R e=0)$ were also performed.

Two inner cylinders with different shapes were used. One is a simple circular cylinder, while the other is a cylinder with two spiral fins as shown in the middle figure of Fig. 1 . The spiral fins have a square cross-section with a side of $t=$ $5 \mathrm{~mm}$, and are attached at an angle of $\theta=45^{\circ}$ to the cylinder. In the present paper, the inner cylinder is rotating in a counter-clockwise direction as viewed from above. With attaching the spiral fins, the flow in the region close to the fins is pushed up.

The position of the inlet of the aeration was varied between $r / d=0.2-0.8$, where $r$ denotes the radial distance from the inner cylinder as shown in Fig.1. By varying the position, the path and floating speed of the bubbles change.

\subsection{Experimental methods of cultivation}

We used cyanobacterium Synechocystis sp. PCC 6803 for cultivation, (Fig. 2), which is one of the most popular cyanobacterium for metabolic engineering studies based on genome information (Kaneko et al., 1996). We used a BG-11 liquid medium (Rippka, 1988) and the bioreactor, which were sterilized using an autoclave unit (ES-315, TOMY SEIKO CO., LTD.). Cyanobacteria cells were pre-cultivated until the suspension became visibly blue-green in $50 \mathrm{ml}$ of BG-11 medium in a glass tube bubbled with air containing $1 \%(\mathrm{v} / \mathrm{v}) \mathrm{CO}_{2}$ at $30{ }^{\circ} \mathrm{C}$. $30 \mathrm{ml}$ of pre-cultivated cells was transferred to $720 \mathrm{ml}$ of new BG-11 medium in the bioreactor and the main cultivation started in the same air and temperature conditions. The light was irradiated from one side of the reactor with an intensity of $20 \mu \mathrm{E} \mathrm{m} \mathrm{s}^{-1}$, which was supplied by two white fluorescent lamps (FL20SW, NEC). The flow rate of the air is about 20 bubbles per 5 seconds. This amount of aeration was empirically determined, and is also sufficient for the development of the algae. However, to clarify the optimal cultivation conditions, the effects of the amount of aeration on the growth rate also have to be investigated in the future work.

To evaluate the growth rate of cyanobacteria, $1 \mathrm{ml}$ of the cell suspension in the reactor was sampled every 24 hours. Then, the cyanobacterial cells were precipitated by centrifugation and the culture medium was removed. $1 \mathrm{ml}$ of methanol was added to the cells and cells were vigorously mixed to extract the chlorophylla, which is the major light-harvesting pigment of cyanobacteria. Then once again the cells were precipitated by centrifugation. The amount of chlorophylla in the supernatant was measured by the absorbance at a wavelength of $665 \mathrm{~nm}$ using a spectral photometer (V-650, JASCO Corporation).

The development of the cyanobacteria in the case with fins $(R e=4000, r / d=0.5)$ is shown in the right figure of Fig. 2 as an example. The time variation of the concentration of chlorophyll was fitted by a logistic equation (Verhulst, 1838) as shown in Eq. (1), where the variation is represented by the S-shaped curve and the gradient of the curve presents the growth rate of living things (Vadasz et al., 2001). The slope at the inflection point is evaluated as the growth rate in the present paper.

$$
y=\frac{\alpha}{1+\beta \exp (-\gamma x)}
$$

\subsection{Measurement of flow fields and behaviors of bubbles}

To clarify the effects of the flow fields in the bioreactor on the growth rate, Particle Image Velocimetry (PIV) was performed as shown in Fig.3. To suppress the refraction of the laser sheet, the bioreactor was placed in a water tank, where a semiconductor laser (Diode-pumped solid-state laser, 532nm) and a high-speed camera (FASTCAM-512PCI 2K, PHOTRON LIMITED) were used. The light from the laser becomes a laser sheet by a cylindrical lens (smc PENTAX-FA $50 \mathrm{~mm}$ F2.8 Macro, RICOH IMAGING COMPANY, LTD). The thickness of the laser sheet was $1 \mathrm{~mm}$.

The image captured by the camera has a size of 512 pixels $\times 512$ pixels, and the resolution estimated using the calibration plate was $0.164 \mathrm{~mm} / \mathrm{pixel}$. The exposed time and frame rate of the camera are $16 \mathrm{sec}$ and $500 \mathrm{~Hz}$, respectively. This frame rate was adjusted so as not to make the particles go out of the laser sheet. To perform the analysis of PIV, a cross-correlation function was computed with an interrogation region of 16 pixels $\times 16$ pixels.

The diameter of the bubbles and time taken for the floating of the bubbles from the lower wall to the upper surface 
of the suspension were also measured by the same high-speed camera. For each case, ten tests were performed and the values were averaged. The bubbles are deformed and sometimes become flattened during the floating, although breakup or coalescence does not occur. The diameter of bubbles was measured when the shape of the bubbles become close to a sphere and the number mean diameter for ten tests was clarified to be $D=4.7 \mathrm{~mm}$ for the diameter of the inlet of the aeration of $3 \mathrm{~mm}$. Also, the diameter of the inlet of the aeration was preliminarily changed from $1 \mathrm{~mm}$ to 5 $\mathrm{mm}$, and the time taken for the floating was measured. As a result, the floating for $3 \mathrm{~mm}$ was the longest and considered to be best for a solution of $\mathrm{CO}_{2}$ bubbles into the suspension. Therefore, the results of the inlet of $3 \mathrm{~mm}(D=$ $4.7 \mathrm{~mm}$ ) are discussed in the present paper.

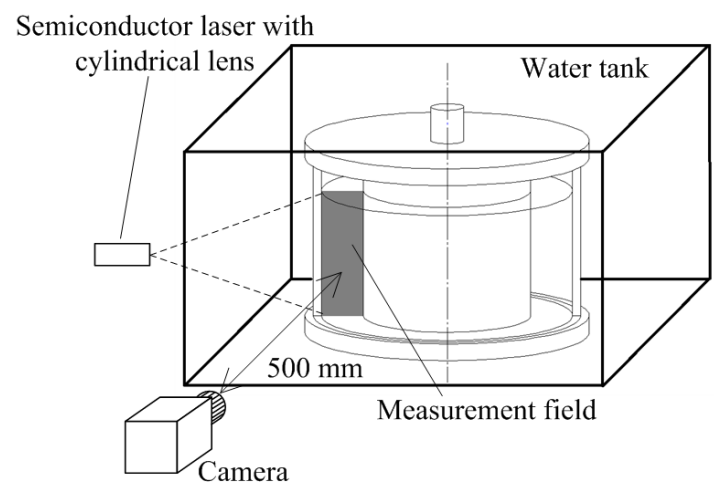

Fig. 3 Schematic overview of particle velocity measurement with a semiconductor laser and a high-speed camera. To suppress the refraction of laser sheet, the bioreactor is placed in a water tank.

\subsection{Numerical methodologies}

To preliminarily evaluate the effects of the position of the inlet for aeration from the inner cylinder, numerical flow simulations (FrontFlow/blue ver.7.2) and a tracking of the bubbles were performed. To reduce the computational cost, Large Eddy Simulations (LESs) were performed for the flow simulations, where the governing equations for an incompressible flow are spatially filtered continuity and Navier-Stokes equations. The dynamic Smagorinsky model (Germano, 1991) with modification by Lilly (Lilly, 1992) was used to account for the effects of subgrid-scale (SGS) stresses. With respect to time integration, an implicit Crank-Nicoloson scheme was used. The spatial discretization is based on the Finite Element Method (FEM). The implemented algorithms have second-order accuracy both in time and space. The Fractional-Step (FS) method was employed to solve the pressure equation. It has been clarified that turbulent flows are successfully captured by this code (Guo et al., 2006).

In the present flow simulations, the hexahedral grids were used, where the numbers of grid points in radial and circumferential directions are 51 and 320 points, respectively, except in the region of the fins. The number in the height direction is 151 points. Also, 15 and 11 grid points are used for the breadth and thickness for the cross-section of the fins. Total grid numbers are 2.3 and 2.8 million for the cases with and without fins, respectively. The simulations were conducted in the rotating system, where non-slip conditions are adopted on the inner cylinder and the other boundaries including the surface at the top are moving walls. In the post-process, the predicted flow field was converted to the inertial system. Although the correct prediction of the effects of free surface at the top on the flow is a future work, the occurrence of a large-scale vortex in the case with the fins on the inner cylinder is correctly captured in this simulation as discussed in subsection 3.1 .

The tracking of the bubbles were also computed by using the flow field predicted by the above-mentioned methods. Referring to the literatures (Ushijima, 2004, and Akiyama et al., 2012), the following Eq. (2) was used, where the Cartesian system is used and the $z$ direction is oriented against the direction of the gravity.

$$
\rho_{\mathrm{D}} \frac{\pi D^{3}}{6} \frac{d \boldsymbol{u}_{\mathrm{D}}}{d t}=\boldsymbol{F}_{\mathrm{A}}+\boldsymbol{F}_{\mathrm{B}}+\boldsymbol{F}_{\mathrm{D}}+\boldsymbol{F}_{\mathrm{L}}
$$

where $\boldsymbol{F}_{\mathbf{A}}, \boldsymbol{F}_{\mathbf{B}}, \boldsymbol{F}_{\mathbf{D}}$ and $\boldsymbol{F}_{\mathbf{L}}$ mean additive mass, buoyancy, drag and lift forces, respectively. Also, $\rho_{\mathrm{D}}$ and $\boldsymbol{u}_{\mathrm{D}}$ are the density and velocity of the bubbles. The additive mass was used with coefficient $C_{\mathrm{M}}$ of 0.5 , referring to Magnaudet $e t$ al. (Magnaudet et al., 1995). To compute the drag forces, the drag coefficient of sphere, $C_{D}$, was estimated referring to 
the literature (Clift et al., 2005). In this tracking, one bubble was taken into consideration because the coalescence of bubbles was not observed in the experiments. However, the effects of the wake of one bubble on another bubble are possibly important, and the investigation of these effects is also a future work. Also, to confirm the validity of the tracking methodology, the experiments of falling of an iron ball in silicon oil and corresponding tracking simulations were preliminarily performed. As a result, it was presented that the track of the ball was correctly predicted by the present method.

\section{Results}

\subsection{Relationship between position of aeration and time for floating of bubbles}

Figure 4 shows the measured and predicted flow fields with and without a fin. In the measurement, the flow close to the outer cylinder was difficult to measure by the refraction of the laser sheet. As shown in the figure, the upward velocity occurs near the inner cylinder and the downward velocity occurs near the outer cylinder in the case with the fins. The predicted upward and downward velocity was relatively smaller than those measured. The reason of this difference is possibly the lack of resolution of the computational grid or the treatment of the upper free surface. However, the present computational results successfully predict the occurrence of a large-scale vortex in the annular gap of the cylinders, which was also observed in the measured results. Therefore, it has been concluded that it is possible to preliminarily evaluate the behaviors of the bubbles. Figure 4 shows that three vortices occur in the case without the fins where the inner cylinder abruptly starts rotating at the determined speed. Because the way of the acceleration of the inner cylinder possibly affects the number of the vortices (Nakamura et al., 1988), the investigation of those effects is a future work,.

The left figure of Fig. 4 shows the contours of vertical velocity and iso-surfaces of $\Delta p$ in an instantaneous flow field. This figure shows that vortices are shed from the leading and trailing edges of the fins. Also the region of the upward velocity appears in the wake of the fins. This upward velocity contributes to the occurrence of the above-mentioned large-scale vortex.
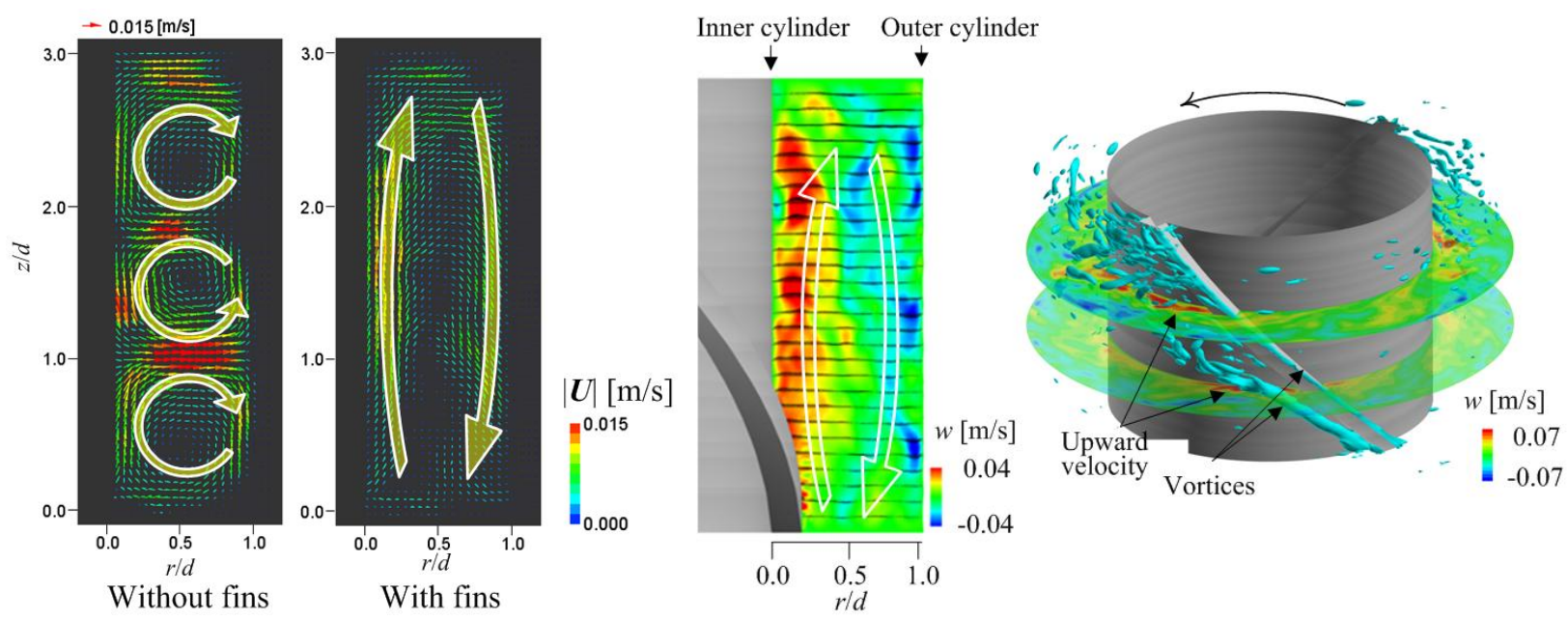

Fig. 4 Measured and predicted flow fields. The left figure shows mean velocity vectors for the cases with and without fins $(R e=4000)$. This figure shows that three vortical structures occur in the case without fins while a large-scale vortex occurs in the case with fins. The middle figure shows the predicted contours of vertical velocity for the case with fins, where the large-scale vortex occurs like in the measured flow field. The right figure shows the predicted instantaneous contours of vertical velocity and iso-surfaces of $\Delta p /\left(\rho U_{0}{ }^{2} / d^{2}\right)=20$.

Figure 5 shows the predicted and measured effects of the position of the aeration on the time taken for the floating of bubbles from the lower wall to the upper surface, $T$, in the bioreactor with the fins, where the floating times for the position of aeration with different circumferential angles from the position of the fins were averaged 10 times and the bars represent these maximum and minimum values in the predicted results. As shown in this figure, the time varies by the angle of the position of aeration from the fin. The difference between the predicted and measured results becomes larger at a position close to the inner and outer cylinders. In the experiments, when the position of the aeration is close to the cylinders, the sphere was sometimes deformed by the walls of the cylinders, while the bubbles were assumed to 
be a sphere in the computation. This is possibly one of the reasons for the difference between the predicted and measured results. Also, in both the predicted and measured results, the time increases for the position of aeration closer to the outer cylinder. This is because the downward velocity close to the outer cylinder as mentioned above makes the floating of the bubbles slow down.

In the present experiment, the breakup or coalescence of the bubbles does not occur. Also, trapping phenomena observed in the literatures (Climent et al., 2007, and Atkhen et al., 2000) was not observed in the present experiment. The effects of the accumulation are possibly smaller with respect to the buoyancy effects because the diameter of the bubbles of $D=4.7 \mathrm{~mm}$ is larger than those of the literatures, where the mean floating velocity $\approx 0.25 \mathrm{~m} / \mathrm{s}$ is higher than the rotating speed of about $0.14 \mathrm{~m} / \mathrm{s}$. However, the trapping effects become important when the smaller bubbles are introduced at a higher Reynolds number.
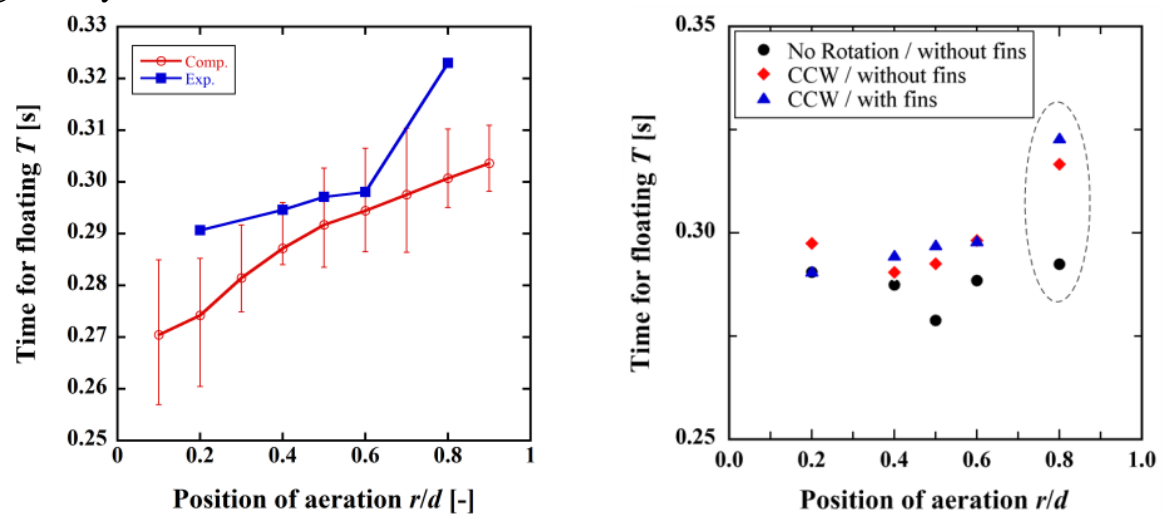

Fig. 5 Relationship between position of aeration and time taken for floating of bubbles $(R e=4000)$. The left figure shows predicted and measured effects of position of aeration on the time taken for the floating of bubbles in the bioreactor with fins, where the red and blue symbols represent computational and measured results, respectively. Both results show that time for floating increases for the position of aeration closer to the outer cylinder. The right figure shows measured times for floating of bubbles for the cases with and without fins in comparison with the case without rotation (no fins). It is clarified that the rotation of the inner cylinder makes the time longer. Moreover, the fins make the time longer particularly at $r / d=0.8$.

\subsection{Effects of rotation and fins of an inner cylinder.}

As shown in Fig. 4, three vortical structures are induced in the annular gap for the case without fins. This result also agrees with the measured result by Nakamura et al. (Nakamura et al., 1988). Meanwhile, the one large-scale vortical structure is induced in the case with fins. This is because the spiral fins push up the fluid in the region close to the inner cylinder and upward velocity is reinforced.

The right figure of Fig. 5 also shows the measured times taken for floating of bubbles for the cases with and without fins $(R e=4000)$, where the time for the case without rotation $(R e=0)$ is also shown. As shown in this figure, the time becomes longer by rotating the inner cylinder. The longer time means that $\mathrm{CO}_{2}$ in the aeration is dissolved more into the suspension and the algae have a greater opportunity to absorb $\mathrm{CO}_{2}$. Also, the figure shows that the fins make the time much longer particularly at $r / d=0.8$ (position close to the outer cylinder). This is because the large-scale vortex induces the downward velocity in the region close to the outer cylinder as mentioned before.

Moreover, it has been confirmed by numerical simulations that the maximum shear stress was only increased $8 \%$ by the fins and that the effects of the fins on cell death are considered to be small. Therefore, attaching fins was considered to be an efficient method for cultivation. In the next subsection, the results of cultivation are shown.

\subsection{Results of cultivation}

The left figure in Fig. 6 shows the measured developments of the cyanobacteria by chlorophyll content for the cases with and without fins $(R e=4000)$ in comparison with the case without rotation (with fins), where the position of the aeration is $r / d=0.5$. The cultivation was continued until the development of the algae was relatively slower to perform experiments with as many parameters as possible. The figure presents that the content can be developed to a higher concentration by mixing.

Figure 7 shows the slope at the inflection point of the curve of the development of cyanobacteria, which indicates 
the growth rate. It is shown that the growth rate becomes more intense by mixing and attaching the fins to the inner cylinder. There are two possible reasons for the higher growth rate by mixing. One of the reasons is that the mixing induces the radial and circumferential movements of the cyanobacteria and the time integration of light for the cyanobacteria is likely to be uniform, which can prevent the oversaturation and shortage of light. The other one of the possible reasons is the increase of the time taken for the floating of the bubbles as mentioned before. As the time increases, the $\mathrm{CO}_{2}$ in the bubbles becomes more likely to dissolve into the suspensions and the cyanobacteria have a greater chance to absorb $\mathrm{CO}_{2}$.

The right figure of Fig. 6 shows the effects of the position of the aeration on the development of the cyanobacteria. The cyanobacteria can be developed to a higher concentration at $r / d=0.8$ (with the fins, $R e=4000$ ) in comparison with the case at $r / d=0.5$. Moreover, Fig. 7 shows that the growth rate is the highest at this case $(\operatorname{Re}=4000$, with fins, $r / d=$ 0.8) in all of the cases presented in this paper. As mentioned in subsection 3.2, the time taken for the floating of the bubbles becomes longest in this case. These results also indicate that increasing the time for the floating of bubbles of aeration contributes to higher productivity of the biomass. To show the effects of the fins and the inlet position of aeration more clearly, experiments with longer duration time and more inlet positions are required. However, the results obtained in this research are believed to indicate the importance of the shape of cylinder and the way of aeration in the design of the bioreactor with a Taylor vortex flow.
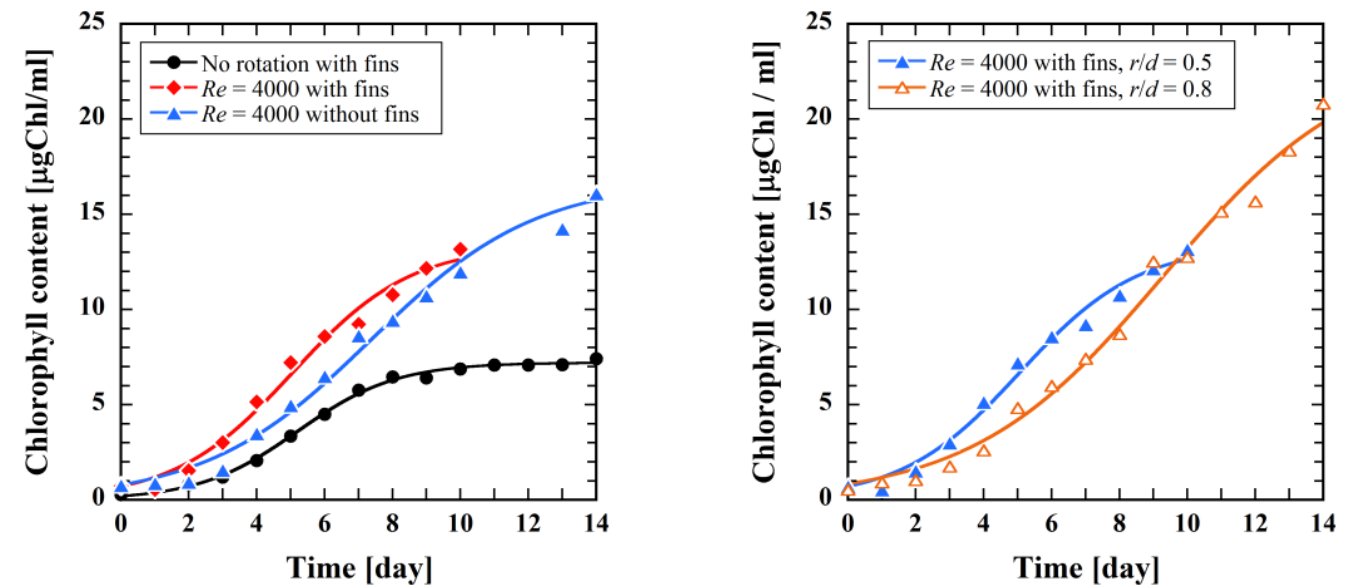

Fig. 6 Measured developments of cyanobacteria. The left figure shows the cases with and without fins $(R e=$ 4000) in comparison with the case without rotation (with fins), where the position of aeration is $r / d=0.5$. The figure shows that the cyanobacteria can be developed to a higher concentration and the slope becomes more intense by mixing. The right figure shows the effects of the position of aeration on the developments. It is clarified that the cyanobacteria can be developed to a much higher concentration at $r / d$ $=0.8$.

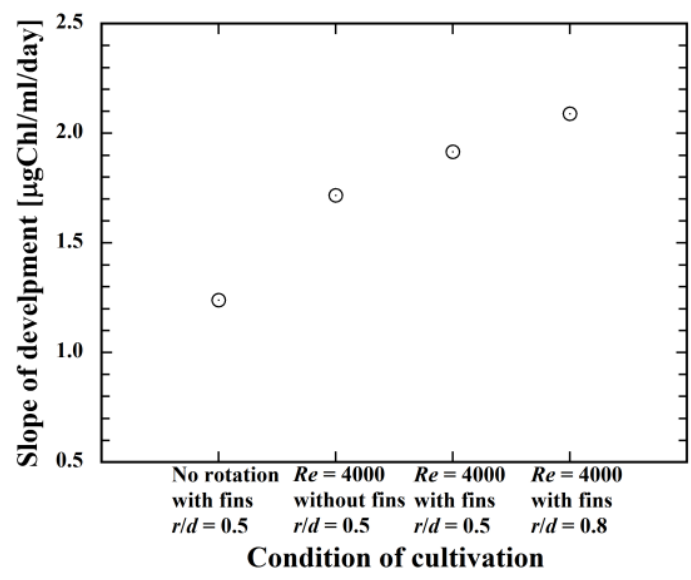

Fig. 7 Measured growth rate of the cyanobacteria for the cases with and without fins $(\mathrm{Re}=4000)$ in comparison with the case without rotation (with fins). The figure shows that the growth rate becomes more intense by mixing and attaching the fins. Also, the growth rate for the case at the position of aeration of $r / d=0.8(R e$ $=4000 . r / d=0.8)$ is the most intense in all of the cases. 


\section{Conclusion}

In order to clarify the effects of the behaviors of the bubbles of aeration on the productivity of the biomass, experiments including cultivation of cyanobacteria and computation were performed utilizing a bioreactor with a Taylor vortex flow in the annular gap of concentric cylinders. Attaching spiral fins to the inner cylinder induces a large-scale vortex in the annular gap instead of the original three vortical structures induced in the case of the simple circular inner cylinder. The downward velocity induced by the large-scale vortex in the region close to the outer cylinder makes the time taken for the floating of bubbles longer when the aeration is performed at the position close to the outer cylinder. At this condition, the cyanobacteria were found to have developed to a high concentration and that the growth rate was also high in the present experiment. These results indicate that the behaviors of the bubbles of aeration are related to the productivity of the biomass.

\section{Acknowledgement}

The present study was supported by JSPS KAKENHI Grant of No. 26820044 and through the Next-generation Supercomputer Strategy Program by the Ministry of Education, Culture, Sports, Science, and Technology of Japan (MEXT). We would like to thank the cooperation by Kazuya Motomura (MEIDENSHA CORPORATION) and Sho Yamamoto (Toyohashi University of Technology).

\section{References}

Akiyama, O., Kato, C., and Kawate, D., Mechanism of Particle Separation in a Cylindrical Cyclone Separator (2nd Report, Validation of Lagrangian Particle Tracking Method and Investigation on Detailed Particle Behaviour), Transactions of the Japan Society of Mechanical Engineers Series B, Vol. 78, No.795 (2012), pp.1903-1918 (in Japanese).

Atkhen, K., Fontaine, J. and Wesfreid, J. E., Highly turbulent Couette-Taylor bubbly flow patterns, Journal of Fluid Mechanics, Vol. 422 (2000), pp.55-68.

Barbosa, M. J., Albrecht, M., Wijffels, R. H., Hydrodynamic stress and lethal events in sparged microalgae cultures. Biotechnology and Bioengineering, Vol.83 (2003), pp.112-120.

Benjamin, T. B., Bifurcation phenomena in steady flows in a viscous fluid I. Theory, Proceedings of the Royal Society of London. Series A, Vol.359 (1978), pp.1-26.

Benjamin, T. B., Bifurcation phenomena in steady flows in a viscous fluid II. Experiment, Proceedings of the Royal Society of London. Series A, Vol.359 (1979), pp.27-43.

Benjamin, T. B., Anomalous Modes in the Taylor Experiment, Proceedings of the Royal Society of London. Series A, Vol.377 (1981), pp.221-249.

Bilson, M. and Bremhost, K., Direct numerical simulation of turbulent Taylor-Couette flow, Journal of Fluid Mechanics, Vol. 579 (2007), pp. 227-270.

Clift, R. J., Grace, R. and Weber, M. E., BUBBLES, DROPS, AND PARTICLES (2005), pp.111-113, DOVER PUBLICATIONS, INC.

Climent, E., Simonnet, M., and Magnaudet, J., Preferential accumulation of bubbles in Couette-Taylor flow patterns, Physics of Fluids, Vol.19 (2007), pp.083301-1-083301-12.

Coles, D., Transition in circular Couette flow, Journal of Fluid Mechanics, Vol.21, part 3 (1965), pp. 385-425.

Dong, S., Direct numerical simulation of turbulent Taylor-Couette flow, Journal of Fluid Mechanics, Vol.587 (2007), pp.373-393.

Eriksen, N. T., The technology of microalgal culturing, Biotechnology Letters, Vol.30 (2008), pp.1525-1536.

Fukushima, N., Fushimi, T., Shimura, M., Tanahashi, M., and Miyauchi, T., Coherent Fine-Scale Eddies near Inner and Outer Walls in Turbulent Taylor-Couette Flow at High Reynolds Number, Nagare, Vol.29 (2010), pp.447-449 (in Japanese).

Germano, M., Piomelli, U., Moin, P. and Cabot, W. H., A dynamic subgrid-scale eddy viscosity model, Physics of Fluids, A3, No.7 (1991), pp.1760-1765.

Gorman, M. and Swinney, H. L., Spatial and temporal characteristics of modulated waves in the circular Couette system, Journal of Fluid Mechanics, Vol.117 (1982), pp.123-142.

Grobbelaar, J. U., Physiological and technological considerations for optimizing mass algal cultures, Journal of Applied Phycology, Vol.12 (2000), pp.201-206. 
Guo, Y., Kato, C. and Yamade, Y., Basic features of the fluid dynamics simulation software FrontFlow/Blue, SEISAN KENKYU, Vol.58, No.1 (2006), pp.11-15.

He, W., Tanahashi, M. and Miyauchi, T., "Direct numerical simulation of turbulent Taylor-Couette flow with high Reynolds number”, European Turbulence Conference, Vol.117 (2007), pp.215-217.

Kaneko T, Sato S, Kotani H, Tanaka A, Asamizu E, Nakamura Y, Miyajima N, Hirosawa M, Sugiura M, Sasamoto S, Kimura T, Hosouchi T, Matsuno A, Muraki A, Nakazaki N, Naruo K, Okumura S, Shimpo S, Takeuchi C, Wada T, Watanabe A, Yamada M, Yasuda M, Tabata S., Sequence analysis of the genome of the unicellular cyanobacterium Synechocystis sp. strain PCC6803. II. Sequence determination of the entire genome and assignment of potential protein-coding regions. DNA Research, Vol.3 (1996), pp.185-209.

Kawai, H., Development of a Mixing Device and Photosynthetic Bioreactor with a Taylor Vortex Flow (Velocity Measurement in Solid-Liquid flows with the Ultrasound Velocity Profiler), The Japanese Society for Multiphase Flow, Vol.24, No.3 (2010), pp.275-281 (in Japanese).

Kliphuis, A. M. J., Winter, Lenneke, W., Vejrazka, C., Martens, D. E., Janssen, M., and Wijffels, R. H., Biotechnology Progress, Vol.26, No.3 (2010), pp.687-696.

Lilly, D. K., A proposed modification of the Germano subgrid-scale closure method, Physics of Fluids, A4, No.3 (1992), pp.633-635.

Magnaudet, J., Rivero, M., and Fabre, J., Accelerated flows past a rigid sphere or a spherical bubble. Part 1. Steady straining flow, Vol.284 (1995), pp.97-135.

Miller, R. L., Fredrickson, A. G., Brown, A. H., Tsuchiya, H. M., Hydromechanical method to increase efficiency of algal photosynthesis, Industrial and Engineering Chemistry Process Design and Development, Vol.3 (1964), pp.134-143.

Nakamura, I., Toyo, Y., Yamashita, S., and Ueki, Y., An Experiment of Taylor-Vortex Flow on a Small Aspect Ratio (1st Report, Comparison Between Asymmetric and Symmetric Condition), Transactions of the Japan Society of Mechanical Engineers Series B, Vol. 54 (1988), pp.1898-1905 (in Japanese).

Pulz, O., Photobioreactors: production systems for phototrophic microorganisms. Applied Microbiology and Biotechnology, Vol.57 (2001), pp.287-293.

Rippka, R, Isolation and purification of cyanobacteria. Methods in Enzymololy, Vol.167 (1988), pp.3-27.

Taylor, G. I., Stability of a Viscous Liquid contained between Two Rotating Cylinders, Philosophical Transactions of the Royal Society of London A, Vol. 223 (1923), pp.289-347.

Ushijima, T., Dispersion and Mixing of Small Particles, Droplets and Bubbles in Turbulent Flows, Nagare, Vol.23 (2004), pp.191-201 (in Japanese).

Vadasz, A.S., Vadasz, P., Abashar, M.E., and Gupthar, A.S., Recovery of an oscillatory mode of batch yeast growth in water for a pure culture, International Journal of Flood Microbiology, Vol.71 (2001), pp.219-314.

Verhulst, P. F., Notice sur la loi que la population suit dans son accroissement. Correspondance Mathematique et Physique Publee par A. Quetelet (Brussels), Vol.10 (1838), No.113-121. 